\title{
GM-CSF-encoding Oncolytic Adenovirus CGTG-102
}

National Cancer Institute

\section{Source}

National Cancer Institute. GM-CSF-encoding Oncolytic Adenovirus CGT G-102. NCI

Thesaurus. Code C98287.

A recombinant, oncolytic serotype 5/3 capsid-modified adenovirus encoding the immunostimulatory cytokine granulocyte-macrophage colony-stimulating factor (GMCSF) with potential antineoplastic activity. Upon administration, the oncolytic adenovirus selectively infects and replicates in tumor cells, which may result in tumor cell lysis. Synergistically, GM-CSF (sargramostim) expressed by the oncolytic adenovirus enhances antigen presentation, promotes natural killer (NK) cell-mediated killing and causes a cytotoxic T cell (CTL) response against tumor cells harboring the oncolytic adenovirus, resulting in an immune-mediated tumor cell death. CGT G-102 is designed to replicate only in cells with defects in the p16/Rb/E2F pathway, attributed to a mutation common in many solid tumors. Replacement of the Ad5 capsid protein knob with a knob domain from serotype 3 causes higher transduction in cancer cells as compared to normal cells. 